\title{
Charge Regulation of Colloidal Particles: Theory and Simulations
}

\author{
Amin Bakhshandeh, ${ }^{1, *}$ Derek Frydel, ${ }^{2, \dagger}$ Alexandre Diehl, ${ }^{3, \$}$ and Yan Levin $\oplus^{4, \S}$ \\ ${ }^{1}$ Programa de Pós-Graduação em Física, Instituto de Física e Matemática, \\ Universidade Federal de Pelotas, Caixa Postal 354, CEP 96010-900 Pelotas, Rio Grande do Sul, Brazil \\ ${ }^{2}$ Department of Chemistry, Federico Santa Maria Technical University, Campus San Joaquin, 7820275 Santiago, Chile \\ ${ }^{3}$ Departamento de Física, Instituto de Física e Matemática, Universidade Federal de Pelotas, \\ Caixa Postal 354, CEP 96010-900 Pelotas, Rio Grande do Sul, Brazil \\ ${ }^{4}$ Instituto de Física, Universidade Federal do Rio Grande do Sul, Caixa Postal 15051, \\ CEP 91501-970 Porto Alegre, Rio Grande do Sul, Brazil
}

(Received 24 July 2019; published 15 November 2019)

\begin{abstract}
To explore charge regulation (CR) in physicochemical and biophysical systems, we present a model of colloidal particles with sticky adsorption sites which account for the formation of covalent bonds between the hydronium ions and the surface functional groups. Using this model and Monte Carlo simulations, we find that the standard Ninham and Parsegian (NP) theory of CR leads to results which deviate significantly from computer simulations. The problem with the NP approach is traced back to the use of a bulk equilibrium constant to account for surface chemical reactions. To resolve this difficulty we present a new theory of CR. The fundamental ingredient of the new approach is the sticky length, which is nontrivially related to the bulk equilibrium constant. The theory is found to be in excellent agreement with computer simulations, without any adjustable parameters. As an application of the theory we calculate the effective charge of colloidal particles containing carboxyl groups, as a function of $p \mathrm{H}$ and salt concentration.
\end{abstract}

DOI: 10.1103/PhysRevLett.123.208004

Electrostatic interactions play a fundamental role in physics, chemistry, and biology. The long-range nature of the Coulomb force, however, makes it very difficult to study theoretically [1]. In aqueous systems ions are usually hydrated by water molecules. On the other hand, acids lose a proton, which associates with the water molecule forming a hydronium ion [2]. There are many reactions that are controlled by $p \mathrm{H}$, and the acid-base equilibrium directly influences the functionality of biomolecules. Although $p \mathrm{H}$ can be easily tuned in experiments, it is much more difficult to account for the chemical equilibrium in theoretical and simulation studies [3].

Colloidal particles often have organic functional groups on their surfaces. In aqueous systems these groups dissociate, losing a proton, resulting in a colloidal surface charge [4-7]. The amount of surface charge strongly depends on the $p \mathrm{H}$ of the environment $[8,9]$ and is controlled by the chemical equilibrium between hydronium ions and the functional groups. This process is known as charge regulation (CR) [10-16]. The concept of charge regulation was first described by Linderstrøm-Lang [17-19] and studied theoretically by Ninham and Parsegian. [20]. CR is of fundamental importance in colloidal science [10,21-32] and biophysics [33-39]. It has been applied to explore the stability of electrical double layers $[9,40-45]$ and is of great technological importance in fields as diverse as mineral preparation, agriculture, ceramics, and surface coating [46].
Consider a weak acid $\mathrm{H} A$ in equilibrium with bulk water, $\mathrm{H} A+\mathrm{H}_{2} \mathrm{O} \rightleftarrows \mathrm{H}_{3} \mathrm{O}^{+}+A^{-}$. For dilute solutions the concentration of all species is controlled by the law of mass action, $K_{\mathrm{eq}}=c_{\mathrm{HA}} / c_{A^{-}} c_{\mathrm{H}^{+}}$, where $K_{\mathrm{eq}}$ is the equilibrium constant and $c$ indicates the concentration of each species. Ninham and Parsegian (NP) supposed that the same equilibrium relation will hold for the reactive (acidic) sites on the colloidal surface with the local concentration of hydronium determined by the Boltzmann distribution, $c_{\mathrm{H}^{+}}^{\text {surf }}=c_{\mathrm{H}^{+}}^{\text {bulk }} \exp \left(-\beta q \phi_{0}\right)$ where $\beta=1 / k_{B} T, q$ is the proton charge, and $\phi_{0}$ is the surface electrostatic potential. NP concluded that the effective surface charge of the colloidal particle will be renormalized from its bare value $-q \sigma_{0}$, corresponding to all functional groups being ionized, by the associated protons. Taking into account the surface equilibrium of hydronium through the Langmuir adsorption isotherm, they argued that one can use the usual PoissonBoltzmann (PB) equation to account for the distribution of ions around the colloidal particle, but with the effective renormalized surface charge given by

$$
q \sigma_{r}=-q \sigma_{0}+\frac{K_{\mathrm{eq}} N_{\text {site }} q c_{a} e^{-\beta q \phi_{0}}}{4 \pi a^{2}\left(1+K_{\mathrm{eq}} c_{a} e^{-\beta q \phi_{0}}\right)},
$$

where $N_{\text {site }}$ is the number of ionizable surface groups, $a$ is the colloidal radius, and $c_{a}$ is the bulk concentration of hydronium ions, $c_{a}=10^{-p \mathrm{H}} \mathrm{M}$. Within the NP formalism $K_{\text {eq }}$ is the usual bulk equilibrium constant. If the surface 
groups are strongly acidic $K_{\text {eq }} \rightarrow 0$, all the surface groups are ionized, $q \sigma_{r} \rightarrow-q \sigma_{0}$.

NP theory has been extensively used to study various biological and chemical systems. However, since within the experiment there is always uncertainty about the underlying physical parameters - the surface charge, location of the shear plane, the appropriate equilibrium constant-it is hard to judge the validity of a theory. In this Letter we propose a model of $\mathrm{CR}$ that has no ambiguities of an experimental system and can be solved exactly using computer simulations. With the help of this model, we find that predictions of NP theory deviate significantly from the results of simulations. We then introduce a new theory which agrees perfectly with the simulation data, allowing us to uniquely predict the number of ionized groups and the ionic distribution around a CR colloidal particle, without any adjustable parameters.

The fundamental parameter in the NP theory is the equilibrium constant. We start, then, by showing how the equilibrium constant can be calculated from a microscopic model. To do this we first consider the Baxter model [47] of sticky hard spheres of species $\mathrm{H}$ and $A$. For simplicity we will suppose that both particles have the same diameter, $d=2 r_{\text {ion }}$. The $\mathrm{H}-\mathrm{H}$ and $A-A$ interactions are purely hardcore repulsion, while collisions between $\mathrm{H}$ and $A$ can result in the formation of molecules $\mathrm{H} A$. The $\mathrm{H}-A$ interaction potential is $u=u_{\mathrm{st}}+u_{\mathrm{hc}}$, where the hard core potential is $u_{\mathrm{hc}}=\infty$ for $r<d$ and 0 otherwise. The attractive sticky potential of range $\delta_{r}$,

$$
u_{\mathrm{st}}(r)= \begin{cases}0, & r<d \quad \text { and } \quad r>d+\delta_{r}, \\ -\epsilon, & d \leq r \leq d+\delta_{r},\end{cases}
$$

is used to model the chemical bonding between $\mathrm{H}$ and $A$. The Boltzmann factor for the sticky potential can then be written as $e^{-\beta u_{\mathrm{st}}(r)}=1+\delta_{r}\left(e^{\beta \epsilon}-1\right) \Delta(r)$, where $\Delta(r)$ is [11]

$$
\Delta(r)= \begin{cases}\frac{1}{\delta_{r}}, & d \leq r \leq d+\delta_{r}, \\ 0, & r<d \quad \text { or } \quad r>d+\delta_{r} .\end{cases}
$$

In the Baxter sticky limit, $\delta_{r} \rightarrow 0, \epsilon \rightarrow \infty$, while the sticky length $l_{g} \equiv \delta_{r}\left(e^{\beta \epsilon}-1\right)$ remains constant, the Boltzmann factor reduces to

$$
e^{-\beta u_{\mathrm{st}}(r)}=1+l_{g} \delta(r-d),
$$

where $\delta(r)$ is the Dirac delta function. The sticky length accounts for the strength of covalent bonds between the atoms and will be directly related to the acid ionization constant.

The equation of state can be calculated using either the "physical picture," which takes into account only "atoms" $\mathrm{H}$ and $A$, or an alternative "chemical picture" in which besides the free unassociated particles $\mathrm{H}$ and $A$, there are also present molecules $\mathrm{H} A$. Clearly both approaches must lead to the same equation of state [48]. Within the physical picture the osmotic pressure $P$ can be obtained using the virial expansion [49]:

$$
\begin{aligned}
\beta P= & c_{\mathrm{H}}+c_{A}+B_{\mathrm{HH}} c_{\mathrm{H}}^{2}+B_{A A} c_{A}^{2} \\
& +2 B_{\mathrm{HA}} c_{\mathrm{H}} c_{A}+\mathcal{O}\left(c_{A}+c_{\mathrm{H}}\right)^{3},
\end{aligned}
$$

where $B_{i j}$ are the second virial coefficients [49]:

$$
B_{i j}=2 \pi \int_{0}^{\infty}\left(1-e^{-\beta u_{i j}(r)}\right) r^{2} d r .
$$

For the case $i=j$ the interaction is just the hard sphere repulsion, so that $B_{A A}=B_{H H}=\frac{2}{3} \pi d^{3}$ and $B_{\mathrm{HA}}=$ $\frac{2}{3} \pi d^{3}+\bar{B}_{\mathrm{HA} A}$, where $\bar{B}_{\mathrm{H} A}=2 \pi \int_{d}^{\infty}\left(1-e^{-\beta u_{i j}(r)}\right) r^{2} d r$. On other hand, in the chemical picture there are three species: free $\mathrm{H}$ and $A$, as well as molecules $\mathrm{H} A$. The equation of state can be written in terms of the respective concentrations designated by $c^{*}$, such that $c_{A}^{*}=c_{A}-c_{\mathrm{H} A}^{*}$ and $c_{\mathrm{H}}^{*}=c_{\mathrm{H}}-c_{\mathrm{HA}}^{*}$, and to second order in density is

$\beta P=c_{\mathrm{H}}^{*}+c_{A}^{*}+c_{\mathrm{H} A}^{*}+\frac{2 \pi}{3} d^{3}\left(c_{A}^{*}+c_{\mathrm{H}}^{*}\right)^{2}+\mathcal{O}\left(c_{A}^{*}+c_{\mathrm{H}}^{*}\right)^{3}$

where the fourth term is just the hardcore contribution to the osmotic pressure. In equilibrium $c_{\mathrm{HA}}^{*}=K_{\mathrm{eq}}^{0} c_{\mathrm{H}}^{*} c_{A}^{*}$, where $K_{\text {eq }}^{0}$ is the equilibrium constant. Comparing Eq. (5) and Eq. (7), we obtain

$$
K_{\mathrm{eq}}^{0}=-2 \bar{B}_{\mathrm{H} A}=4 \pi \int_{d}^{\infty}\left(e^{-\beta u_{\mathrm{st}}(r)}-1\right) r^{2} d r .
$$

In the Baxter sticky limit the equilibrium constant simplifies to $K_{\text {eq }}^{0}=4 \pi d^{2} l_{g}$. In this simple calculation our particles $\mathrm{H}$ and $A$ interacted only through a hard core repulsion and a short range attraction. If we are interested in modeling the acidic groups, both $\mathrm{H}$ and A must also carry charge, $\mathrm{H}^{+}$and $A^{-}$. In this case the calculations become much more involved, since the usual virial expansion diverges and instead a certain class of diagrams must be summed together to obtain a convergent result [49]. This leads to a nonanalytic term proportional to $c^{3 / 2}$ in the density expansion. Falkenhagen and Ebeling [50,51] studied this problem in order to account for the formation of Bjerrum pairs in a 1:1 electrolyte solution, and we can extend their results to the particles which in addition to the Coulomb force also interact through a short range sticky potential, for details of the derivation see Supplemental Material (SM)[52]. In our case the equilibrium constant becomes

$$
K_{\text {eq }}=4 \pi d^{2} l_{g} e^{b}+K_{E b},
$$

where $b=\lambda_{B} / d$ and $\lambda_{B}$ is the Bjerrum length $q^{2} / \epsilon_{w} k_{B} T$. The Ebeling equilibrium constant is [53] 


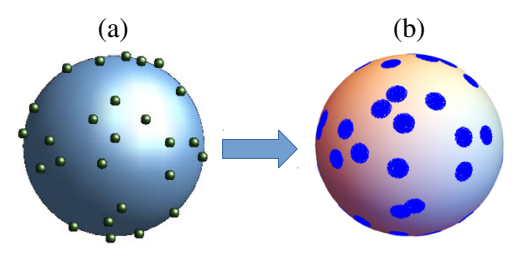

FIG. 1. (a) Representation of a colloidal particle with spherical $A^{-}$sticky site on its surface. (b) Mapping of spherical sticky sites onto disklike surface patches used in the new theory of CR.

$$
\begin{aligned}
K_{E b}= & 8 \pi d^{3}\left\{\frac{1}{12} b^{3}[\operatorname{Ei}(b)-\operatorname{Ei}(-b)]-\frac{1}{3} \cosh b\right. \\
& \left.-\frac{1}{6} b \sinh b-\frac{1}{6} b^{2} \cosh b+\frac{1}{3}+\frac{1}{2} b^{2}\right\},
\end{aligned}
$$

where Ei is the exponential integral function.

We can now explore the validity of NP theory by constructing a simple model of a colloidal particle with $A^{-}$sticky surface groups, see Fig. 1(a). This model can then be studied using Monte Carlo (MC) simulations [54,55]. Knowledge of the equilibrium constant $K_{\text {eq }}$ will also allow us to directly compare the effective colloidal charge and the ionic density profiles obtained using NP theory with the results of MC simulations.

The simulations are performed inside a spherical Wigner-Seitz (WS) cell of radius $R$, determined by the colloidal volume fraction in the suspension, $v=a^{3} / R^{3}$. A colloidal particle of radius $a=100 \AA$ and $Z$ spherical adsorption sites of radius $2 \AA$ and charge $-q$, randomly distributed on its surface, is placed at the center of the simulation cell, see Fig. 1(a). The bare colloidal charge $-Z q$ is the same as the number of adsorption sites. The WS cell also contains hydronium ions at bulk concentration $c_{a}=10^{-p \mathrm{H}}$, derived from the dissociation of a strong acid, as well as $1: 1$ strong electrolyte at concentration $c_{s}$. The hydronium ions interact with the adsorption sites through both the Coulomb and short range Baxter potential with $l_{g}=109.97 \AA$, while all other ions interact only through the Coulomb force. All ions and sticky sites are modeled as hard spheres of radius $r=2 \AA$, with a point charge at the center. Note that the alkali metal cations derived from the dissociation of salt can come as close to the adsorption sites as the hard core repulsion allows; however, since there is no chemical reaction between $\mathrm{Na}^{+}$and carboxyl in watersodium acetate is a very strong electrolyte and is fully dissociated in water-we assume that there is only a Coulomb interaction between alkali metal cations and the surface groups.

To perform the simulations we have used a progressively smaller values of $\delta_{r}$, and larger values of $\epsilon$ to check the convergence to the Baxter sticky limit, see the discussion in the SM. The solvent is treated as a dielectric continuum of permittivity $\epsilon_{w}=80$, with Bjerrum length $\lambda_{B}=7.2 \AA$. If the hydronium ion adsorbs to a sticky site, the site becomes inactive (stickiness is turned off) and no other hydroniums can be adsorbed. This mimics the chemical reaction which takes place at the adsorption (sticky) site. Note that in the simulation the sites are active or inactive depending on whether there is a hydronium ion within the range of the potential $u(r)$, see the SM for more details. We have used $5 \times 10^{6}$ MC steps for equilibration and $10^{5}$ steps for production.

In Fig. 2 we compare the density profiles obtained using the MC simulations with the predictions of NP theory using the bulk equilibrium constant derived in Eq. (9). We see that there is a significant deviation between the theory and simulations, even when only neutralizing hydronium ions are present inside the simulation cell.

We can trace the breakdown of the NP theory to the use of the bulk equilibrium constant to account for the surface chemical reaction. While the particles in the bulk are free to move, the adsorption sites are bound to the surface. This affects the entropic contribution to the adsorption free energy. Furthermore, as can be seen from Eq. (9), the bulk equilibrium constant also includes a contribution from the Coulomb interaction between the two oppositely charged ions that form neutral molecules. On the other hand, the Coulomb interaction is also taken into account in the solution of the $\mathrm{PB}$ equation and is, therefore, counted twice. Finally, the concentration of ions near a strongly charged surface can be so large that the use of concentration instead of activity, might not be justified. In view of these observations, we now propose a different approach to CR.

Let us first imagine that the whole colloidal surface is uniformly sticky. The concentration of ions around the colloidal particle will then satisfy a modified PB (MPB) equation
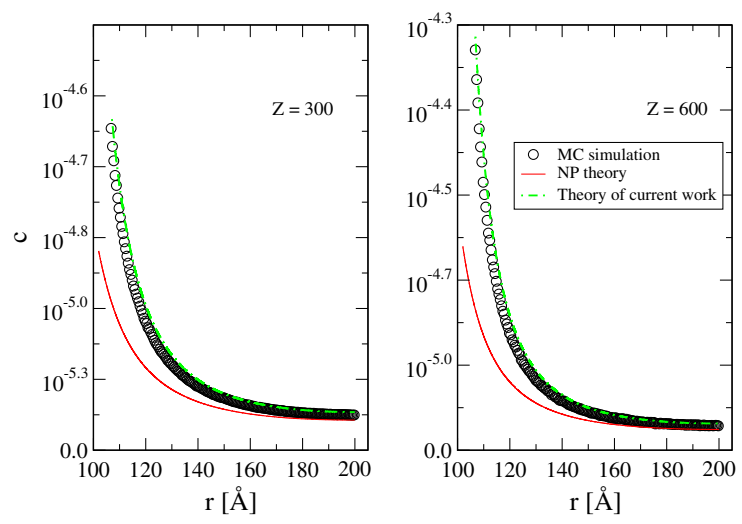

FIG. 2. Density profile of hydronium counterions measured in particles per $\AA^{3}$. Symbols are the simulation data and solid (red) and dashed (green) lines are the predictions of the NP theory and of the theory developed in the present Letter, respectively. The parameters are $a=100 \AA, R=200 \AA$, and $l_{g}=109.97 \AA$. In (a) and (b) the colloidal particles have respectively 300 and 600 active surface sites. 
$\nabla^{2} \phi(r)=-\frac{4 \pi q}{\epsilon_{w}}\left[-\sigma_{0} \delta(r-a)+c_{\mathrm{H}^{+}}(r)+c_{+}(r)-c_{-}(r)\right]$,

where $\sigma_{0}=Z / 4 \pi\left(a+r_{\text {ion }}\right)^{2}$. The ionic concentrations are

$$
\begin{aligned}
c_{\mathrm{H}^{+}}(r) & =c_{a} e^{-\beta[u(r)+q \phi(r)]} \\
c_{+}(r) & =c_{s} e^{-\beta q \phi(r)} \\
c_{-}(r) & =\left(c_{a}+c_{s}\right) e^{\beta q \phi(r)},
\end{aligned}
$$

where the bulk concentration of hydronium is $c_{a}=10^{-p \mathrm{H}} \mathrm{M}$, and $c_{s}$ is the bulk concentration of salt.

Using Eq. (4) we obtain $e^{-\beta[u(r)+q \phi(r)]}=$ $\left[1-l_{g} \delta\left(r-a-r_{\text {ion }}\right)\right] e^{-\beta q \phi(r)}$. The surface concentration of adsorbed ions is then

$$
\sigma_{a}=c_{a} l_{g} e^{-\beta q \phi_{0}},
$$

where $\phi_{0}=\phi\left(a+r_{\text {ion }}\right)$ is the contact electrostatic potential. The net surface charge density is then $q \sigma_{\text {net }}=$ $-q \sigma_{0}+q \sigma_{a}$.

The real colloidal surface, however, is not uniformly sticky, instead hydronium ions can adsorb only at the specific functional groups, see, Fig. 1(a). To understand the role of discreteness of the surface charge [56], let us first consider a much simpler problem. Suppose we have an isolated colloidal particle (no other ions), with $Z$ fully ionized surface groups. How much work must be done to bring a counterion from infinity into contact with one of the surface groups? To answer this question, let us first consider a spherical 2D one component plasma (OCP) of $Z$ charged point particles on a sphere of radius $a$ with a neutralizing uniform background. In a crystal or amorphous state the electrostatic energy of this OCP is $F^{\mathrm{OCP}} \approx-M q^{2} Z^{3 / 2} / 2 \epsilon_{w} a$, where $M=1.106$ is the Madelung constant [1]. On the other hand the OCP energy can also be split into distinct contributions: $F^{\mathrm{OCP}}=Z^{2} q^{2} / 2 \epsilon_{w} a-Z^{2} q^{2} / \epsilon_{w} a+F^{q q}$, where the first term is the self-energy of the neutralizing background, the second term is the interaction of the discrete charges with the background, and the last term is the interaction energy between the discrete charges. This last term is of particular interest to us since it is precisely the electrostatic energy of an isolated colloidal particle with discrete surface groups. Using the expressions above, it can be written as

$$
F^{q q}(Z)=\frac{Z^{2} q^{2}}{2 \epsilon_{w} a}-\frac{M q^{2} Z^{3 / 2}}{2 \epsilon_{w} a} .
$$

The work required to bringing a counterion to the colloidal surface equals to the change in the electrostatic energy $\mu=$ $F^{q q}(Z-1)-F^{q q}(Z)$ or,

$$
\mu \approx-\frac{\partial F^{q q}}{\partial Z}=-\frac{Z q^{2}}{\epsilon_{w} a}+\frac{3 M q^{2} Z^{1 / 2}}{4 \epsilon_{w} a} .
$$

Note that the first term on the right of Eq. (17) is just the usual mean-field interaction energy between a counterion and a uniformly charged sphere $q \phi_{0}$, where $\phi_{0}$ is the "mean-field" surface potential, while the second term is the correction due to discrete nature of the surface charge groups. If the counterion is brought into contact with one of the surface groups, the total work $\varphi_{0}$ is

$$
\beta \varphi_{0}=\beta q \phi_{0}+\frac{3 M \lambda_{B} Z^{1 / 2}}{4 a}-\frac{\lambda_{B}}{d},
$$

where the last term is the direct energy of interaction between the site and the adsorbed counterion. With these insights, we now return to the problem of a colloidal particle inside an electrolyte solution. To simplify the geometry we will map the sticky spherical sites onto sticky circular disk patches of the same effective contact area. Because of the hardcore repulsion only half of the area of a spherical sticky site is available for adsorption, the patches must then have radius $r_{\text {patch }}=\sqrt{2} d$, see Fig. 1(b). Once adsorption takes place, the site becomes inactive, but continues to interact with the other ions through the Coulomb potential. The fact that only part of the colloidal surface is sticky can be taken into account by the renormalization of the sticky length $l_{g} \rightarrow l_{g}^{\text {eff }}=l_{g} \alpha_{\text {eff }}$, where $\alpha_{\text {eff }}$ is the fraction of colloidal surface area occupied by the active sticky patches,

$$
\alpha_{\mathrm{eff}}=\frac{N_{\mathrm{site}}^{\mathrm{act}} r_{\mathrm{patch}}^{2}}{4 a^{2}} .
$$

The number of active sites, $N_{\text {site }}^{\text {act }}$, is determined from Eq. (15) with $l_{g} \rightarrow l_{g}^{\text {eff }}$ and $\phi_{0} \rightarrow \varphi_{0}$, so that $N_{\text {site }}^{\text {act }}=Z-4 \pi a^{2} c_{a} l_{g}^{\text {leff }} e^{-\beta \varphi_{0}}$. The surface electrostatic potential $\varphi_{0}$ is given by Eq. (18), with $\phi_{0}$ now being the mean-field surface electrostatic potential, which must be calculated self-consistently from the solution of the PB equation. The equation $l_{g}^{\text {eff }}=l_{g} \alpha_{\text {eff }}$ and Eq. (19) can now be solved to obtain the effective sticky length

$$
l_{g}^{\mathrm{eff}}=\frac{l_{g} Z r_{\mathrm{patch}}^{2}}{4 a^{2}\left(1+l_{g} c_{a} e^{-\beta \varphi_{0}} \pi r_{\mathrm{patch}}^{2}\right)} .
$$

The effective surface charge density to be used as the boundary condition for the $\mathrm{PB}$ equation is then $q \sigma_{\text {eff }}=-q \sigma_{0}+q l_{g}^{\text {eff }} c_{a} \mathrm{e}^{-\beta \varphi_{0}}$. We now solve the $\mathrm{PB}$ equation with the boundary conditions $\phi^{\prime}\left(a+r_{\text {ion }}\right)=$ $4 \pi q \sigma_{\text {eff }} / \epsilon_{w}$ and $\phi^{\prime}(R)=0$, due to the overall charge neutrality. The calculation is performed numerically using the fourth-order Runge-Kutta method, in which the value of the surface potential $\phi\left(a+r_{\text {ion }}\right)=\phi_{0}$ is adjusted based on the Newton-Raphson algorithm to obtain zero electric field at the cell boundary. In Fig. 3 we compare the ionic density profile obtained using MC simulations and the new theory. 

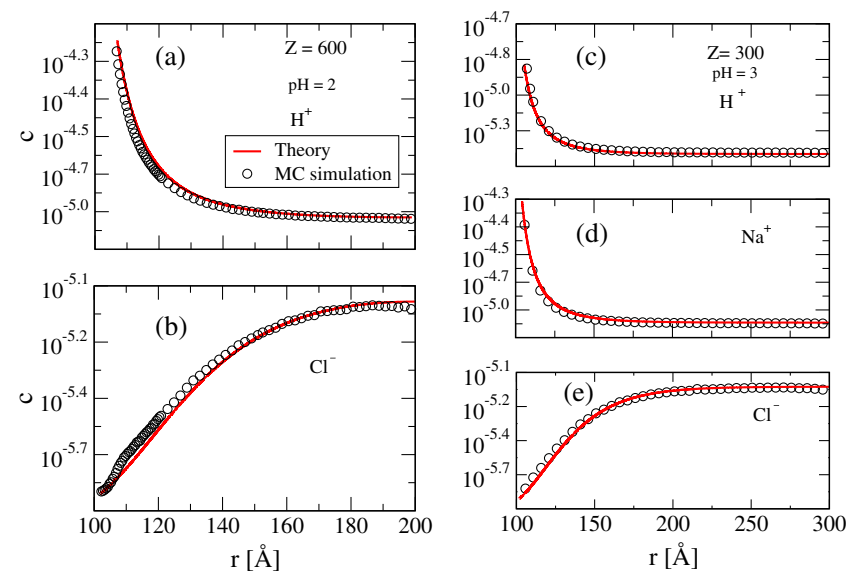

FIG. 3. Comparison between theory (solid lines) and simulation (symbols). Panel (a) is the density profile of hydronium and (b) of $\mathrm{Cl}^{-}$for colloidal particles with $Z=600$ functional groups and volume fraction of $12.5 \%$ in hydrochloric acid at $p \mathrm{H}=2$. Panels (c), (d), and (e) are the hydronium, $\mathrm{Na}^{+}$, and $\mathrm{Cl}^{-}$, density profiles, respectively, for colloidal particles with $Z=300$ functional groups and volume fraction of $3.7 \%$, in a solution of hydrochloric acid of $p \mathrm{H}=3$ and $10 \mathrm{mM}$ of $\mathrm{NaCl}$. The density $C$ is in units of particles per $\AA^{3}$. The sticky length is $l_{g}=109.97 \AA$.

The agreement is excellent, without any adjustable parameters.

Having established the accuracy of the theoretical approach, we now use it to calculate the effective charge of colloidal particles stabilized by surface carboxyl groups with acid ionization constant $K_{a}=1.8 \times 10^{-5} \mathrm{M}$. Note that the equilibrium constant $K_{\text {eq }}$ defined in the present work is the inverse of $K_{a}$. Using $K_{\mathrm{eq}}=1 / K_{a}$ in Eq. (9) allows us to obtain the sticky length $l_{g}$. In Fig. 4 we show the dependence of the effective colloidal charge on the $p \mathrm{H}$ and salt concentration for particles with $Z=600$ functional groups on the surface. In the SM we also plot the behavior of the modulus of the contact electrostatic potential. While $Z_{\text {eff }}$ increases with salt, the modulus of the contact potential and, therefore, the zeta potential, decrease with the salt concentration [57].

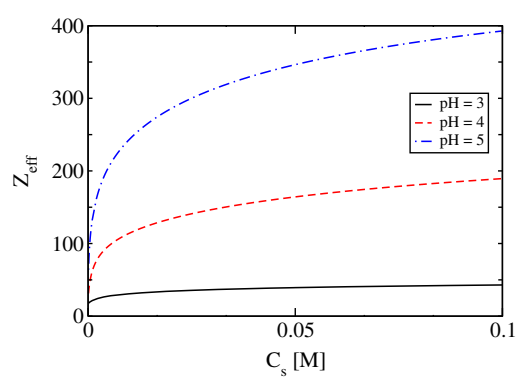

FIG. 4. Effective charge of a colloidal particle of radius $a=$ $100 \AA$ and $Z=600$ carboxyl groups, as a function of salt concentration, in a solution of a given $p \mathrm{H}$ obtained using the new theory of CR. Colloidal volume fraction is $12.5 \%$.
In this Letter we have presented a model of colloidal particle with sticky adsorption sites. Analyzing the thermodynamics of ionic association, we were able to relate the interaction potential between the adsorption sites and hydronium ions with the bulk equilibrium constant. With the help of this model we discovered that existing approaches were not able to quantitatively account for $\mathrm{CR}$, predicting the incorrect value of the colloidal charge and ionic density profiles which deviated significantly from simulations. With the insights gained from the simulations, we were able to introduce a new theory of CR. The next step is to explore the role of CR on the interaction between colloidal particles and the role that it will play in the stability of colloidal suspensions. This should be possible to do by implementing the CR boundary condition analogously to the approach recently employed for metal particles [58].

This work was partially supported by CNPq, CAPES, and the US-AFOSR under Grant No. FA9550-12-1-0438.

*amin.bakhshandeh@ufrgs.br

†derek.frydel@usm.cl

†diehl@ufpel.edu.br

${ }^{\S}$ levin@if.ufrgs.br

[1] Y. Levin, Rep. Prog. Phys. 65, 1577 (2002).

[2] N. Agmon, Chem. Phys. Lett. 319, 247 (2000).

[3] M. D. Baer, J. L. Fulton, M. Balasubramanian, G. K. Schenter, and C. J. Mundy, J. Phys. Chem. B 118, 7211 (2014).

[4] S. Alexander, P. M. Chaikin, P. Grant, G. J. Morales, P. Pincus, and D. Hone, J. Chem. Phys. 80, 5776 (1984).

[5] E. Trizac, L. Bocquet, and M. Aubouy, Phys. Rev. Lett. 89, 248301 (2002).

[6] L. Bocquet, E. Trizac, and M. Aubouy, J. Chem. Phys. 117, 8138 (2002).

[7] P. Wette, H. J. Schöpe, and T. Palberg, J. Chem. Phys. 116, 10981 (2002).

[8] A. Adamson and A. Gast, Chemistry of Surfaces (John Wiley \& Sons, New York, NY, USA, 1997).

[9] T. Markovich, D. Andelman, and R. Podgornik, Europhys. Lett. 113, 26004 (2016).

[10] R. Podgornik, J. Chem. Phys. 149, 104701 (2018).

[11] D. Frydel, J. Chem. Phys. 150, 194901 (2019).

[12] Y. Avni, T. Markovich, R. Podgornik, and D. Andelman, Soft Matter 14, 6058 (2018).

[13] Y. Avni, D. Andelman, and R. Podgornik, Curr. Opin. Electrochem. 13, 70 (2019).

[14] T. Sen and M. Barisik, Sci. Rep. 9, 137 (2019).

[15] M. Blanco Pablo, S. Madurga, F. Mas, and J. Garcés, Polymers 10, 8 (2018).

[16] H. Gokberk Ozcelika and M. Barisik, Phys. Chem. Chem. Phys. 21, 7576 (2019).

[17] K. Linderstrøm-Lang, C.R. Trav. Lab. Carlsberg 15 (1924).

[18] M. Lund and B. Jönsson, Q. Rev. Biophys. 46, 265 (2013).

[19] I. Gitlin, M. Mayer, and G. M. Whitesides, J. Phys. Chem. B 107, 1466 (2003). 
[20] B. W. Ninham and V. A. Parsegian, J. Theor. Biol. 31, 405 (1971).

[21] D. C. Prieve and E. Ruckenstein, J. Theor. Biol. 56, 205 (1976).

[22] S. L. Carnie and D. Y. Chan, J. Colloid Interface Sci. 161, 260 (1993).

[23] S. H. Behrens and M. Borkovec, J. Chem. Phys. 111, 382 (1999).

[24] R. Netz, J. Phys. Condens. Matter 15, S239 (2003).

[25] M. L. Henle, C. D. Santangelo, D. M. Patel, and P. A. Pincus, Europhys. Lett. 66, 284 (2004).

[26] G. Trefalt, S. H. Behrens, and M. Borkovec, Langmuir 32, 380 (2016).

[27] A. Majee, M. Bier, and R. Podgornik, Soft Matter 14, 985 (2018).

[28] A. Lošdorfer Božič and R. Podgornik, J. Chem. Phys. 149, 163307 (2018).

[29] J. E. Hallett, D. A. Gillespie, R. M. Richardson, and P. Bartlett, Soft Matter 14, 331 (2018).

[30] F. Waggett, M. Shafiq, and P. Bartlett, Colloids Interfaces 2, 51 (2018).

[31] G. Trefalt, H. B. Sven, and M. Borkovec, Langmuir 32, 380 (2016).

[32] N. Boon and R. van Roij, J. Chem. Phys. 134, 054706 (2011).

[33] M. Lund and B. Jönsson, Biochemistry 44, 5722 (2005).

[34] M. Heinen, T. Palberg, and H. Löwen, J. Chem. Phys. 140, 124904 (2014).

[35] C.-Y. Leung, L. C. Palmer, S. Kewalramani, B. Qiao, S. I. Stupp, M. Olvera de la Cruz, and M. J. Bedzyk, Proc. Natl. Acad. Sci. U.S.A. 110, 16309 (2013).

[36] G. S. Longo, M. Olvera de la Cruz, and I. Szleifer, Macromolecules 44, 147 (2011).

[37] M. Tagliazucchi, M. O. de la Cruz, and I. Szleifer, Proc. Natl. Acad. Sci. U.S.A. 107, 5300 (2010).

[38] R. Govrin, Biophys. J. 114, 224a (2018).

[39] R. A. Hartvig, M. van de Weert, J. Østergaard, L. Jorgensen, and H. Jensen, Langmuir 27, 2634 (2011).
[40] R. Podgornik and V. Parsegian, Chem. Phys. 154, 477 (1991).

[41] T. Markovich, D. Andelman, and R. Podgornik, Europhys. Lett. 120, 26001 (2017).

[42] D. Leckband and J. Israelachvili, Q. Rev. Biophys. 34, 105 (2001).

[43] F. L. B. da Silva and B. Jönsson, Soft Matter 5, 2862 (2009).

[44] A. Smith, P. Maroni, M. Borkovec, and G. Trefalt, Colloids Interfaces 2, 65 (2018).

[45] N. Adžič and R. Podgornik, Phys. Rev. E 91, 022715 (2015).

[46] R. J. Hunter, Foundations of Colloid Science (Oxford University Press, New York, 2001).

[47] R. Baxter, J. Chem. Phys. 49, 2770 (1968).

[48] T. L. Hill, Statistical Mechanics: Principles and Selected Applications (Dover Publications, New York, 1987).

[49] D. A. McQuarrie, Statistical Thermodynamics (Harper and Row, New York, 1973).

[50] H. Falkenhagen and W. Ebeling, Equilibrium Properties of Ionized Dilute Electrolytes (Academic Press, New York, 1971).

[51] Y. Levin and M. E. Fisher, Physica (Amsterdam) 225A, 164 (1996).

[52] See Supplemental Material at http://link.aps.org/ supplemental/10.1103/PhysRevLett.123.208004 for derivation of Eq. (9); convergence to the baxter limit; comparison with the Ninham-Parsegian theory (np); contact potentials.

[53] W. Ebeling, Z. Phys. Chem. (Leipzig) 238, 400 (1968).

[54] D. Frenkel and B. Smith, Understanding Molecular Simulation (Academic Press, New York, 1996).

[55] M. P. Allen and D. J. Tildesley, Computer Simulations of Liquids (Oxford University Press, Oxford, 1987).

[56] Y. S. Jho, S. A. Safran, M. In, and P. A. Pincus, Langmuir 28, 8329 (2012).

[57] S. H. Behrens, D. I. Christl, R. Emmerzael, P. Schurtenberger, and M. Borkovec, Langmuir 16, 2566 (2000).

[58] A. P. dos Santos and Y. Levin, Phys. Rev. Lett. 122, 248005 (2019). 